\title{
Evaluation of Pulsed Electromagnetic Field in the Management of Osteoarthritis using Electromyography - An Interventional study
}

\author{
Sajuni $\mathbf{I}^{\mathbf{1}}$, Karthika $\mathbf{M}^{\mathbf{2}}$, Ezhilnila $\mathbf{S}^{\mathbf{3}}$, M R Lalitha ${ }^{\mathbf{4}}$, Vishnuprasaath $\mathbf{S}^{\mathbf{5}}$ \\ ${ }^{I}$ Assistant Professor, Department of Physiology, Government Pudukkottai Medical college \& Hospital, Pudukkottai. \\ ${ }^{2}$ Assistant Professor, ${ }^{3}$ Assistant Professor, ${ }^{4}$ Post Graduate, Department of Physiology, \\ Dhanalakshmi Srinivasan Medical college \& Hospital, Perambalur, Tamil Nadu, India. \\ ${ }^{5}$ Tutor, ESIC Medical college, K.K.Nagar, Chennai, Tamil Nadu, India.
}

\begin{abstract}
Knee pain is one of the most frequent musculoskeletal problems among the middle-aged and elderly people and 'Osteoarthritis' is the widespread clinical pathology of the knee. Objective: The present study was conducted to evaluate whether low frequency Pulsed Electro Magnetic Field (PEMF) therapy is effective in patients with osteoarthritis knee using surface Electromyography (sEMG) of vastus medialis. Methods: The participants were subjected to Pulsed Electromagnetic Field Therapy using the PULSATRON instrument designed by Madras Institute of Magnetobiology, Anna nagar. They were given PEMF therapy of $10 \mathrm{~Hz}$ for 60 minutes/ day for 21 days with a break after every 6 days. The participants of the study were subjected to Electromyography of vastus medialis using the MEDICAID PHYSIOPAC machine. The findings are compared before and after the duration of PEMF therapy. Results: The study shows that Low frequency PEMF $(10 \mathrm{~Hz})$ can be used as a treatment modality for Osteoarthritis. PEMF therapy, when given for a sufficient time (21 days) can improve the stiffness and the power of muscles as seen by the increase in amplitude of electromyography. The mean amplitude of sEMG in vastus medialis was found to be significantly increased $(p<0.001)$ in the study group following the Pulsed Electro Magnetic Field (PEMF) therapy.
\end{abstract}

Keywords: Low frequency PEMF; sEMG in vastus medialis.

\section{Introduction}

Knee pain is one of the most frequent musculoskeletal problems among the middle-aged and elderly people and 'Osteoarthritis' is the widespread clinical pathology of the knee. Osteoarthritis causes degradation of joints, articular cartilage, and subchondral bone [1]. Apart from the knee joint, it also involves hip, spine and hand joints. About $40 \%$ of the Indian Population around the age of 70 suffer from osteoarthritis among which $2 \%$ of the population experience crucial pain and disability [2]. Females are found to be affected more severely with osteoarthritis with large number of joints involved particularly the knee and hand [3].

Osteoarthritis can be broadly classified into two main categories - Primary osteoarthritis and Secondary osteoarthritis [4]. The Primary form of osteoarthritis occurs due to an idiopathic etiology. The secondary forms of osteoarthritis occur because of some underlying pathology. The common etiologies include: Congenital, Post surgery, injury, Endocrine, Metabolic, Rheumatologic and Neurological.

Patients of osteoarthritis knee usually present with knee pain, stiffness, swelling of the joint, cracking or grinding noise with joint movement and decreased function of the joint. A longstanding osteoarthritis knee if left untreated may lead to severe functional disability. The limitations are mainly due to the cartilage defect, erosion and fusion of the joint and stiffness of the muscle.

eISSN: 2523-6709

pISSN: 2523-6695

DOI: $10.31878 /$ ijcrpp.2020.42.03
The diagnosis of osteoarthritis is usually based on history, clinical examination, and X-rays. Most of the treatments at present are focusing on pain reduction, improving the joint stiffness and maintenance of joint function. Prompt treatment is necessary to avoid these complications.

The patients of osteoarthritis are functionally inferior when compared to the control population of the same age and sex [5]. The primary reason for this decreased functional capability of osteoarthritis patients is the loss of muscular strength of the lower extremities which may be due to muscular atrophy and inhibition [6-9]. The assessment of the strength of quadriceps is an important task in these patients, which is studied by Electromyography (EMG). Electromyography is an experimental technique mainly involved with the development, recording and analysis of myoelectric signals [10]. Electromyography has both diagnostic and prognostic value.

Pulsed Electromagnetic Field (PEMF) is a modern therapeutic device used in treating many clinical conditions. Though the clinical application of PEMF is still under controversy, it is being widely used as a treatment modality over the past two decades and has shown to decrease pain, inflammation, and stiffness in patients with osteoarthritis [11]. The mechanism behind this is the increase in blood supply to peri-articular compartment due to synthesis of nitric oxide [12].

This study focuses on evaluating whether the low frequency and low intensity Pulsed Electromagnetic Field (PEMF) therapy is effective in reducing osteoarthritis pain by evaluation of quadriceps function by Electromyography (EMG) before and after administration of Pulsed Electro Magnetic Field Therapy.

Correspondence: Dr. Karthika. M, Assistant Professor, Department of Physiology, Dhanalakshmi Srinivasan Medical college \& Hospital, Perambalur, Tamil Nadu, India. Email: mkarthikambbs@gmail.com 


\section{Materials and Methods}

An interventional study was conducted in the Institute of Physiology and Experimental Medicine, Madras Medical College in collaboration with the Institute of Geriatrics, Rajiv Gandhi Government General Hospital, Chennai from April 2015 to March 2016. Fifty patients between 50 to 70 years of age with osteoarthritis knee having symptoms for at least one year duration were included in the study after obtaining Ethical approval from Institutional Ethics Committee (IEC), Madras Medical College, Chennai. Patients with chronic disorders were excluded from the study.

\section{Methodology:}

The participants of the study were subjected to Electromyography of vastus medialis using the MEDICAID PHYSIOPAC machine. The power of the muscle was assessed by using surface electromyography. The participants were subjected to Pulsed Electromagnetic Field Therapy using the PULSATRON instrument designed by Madras Institute of Magnetobiology, Anna Nagar. They were given PEMF therapy of $10 \mathrm{~Hz}$ for 60 minutes/ day for 21 days with a break after every 6 days (protocol designed by Madras Institute of Magnetobiology). After the Pulsed Electromagnetic Field Therapy, the subjects again underwent the study of electromyography. The findings are compared before and after the duration of PEMF therapy.

Statistical Analysis: After collection, data were checked for consistency and completeness. Then the data was entered in database Statistical Package for the Social Sciences (SPSS) software version 21. The Paired Student's t test was carried out to compare the mean of variables before and after administration of Pulsed Electro Magnetic Field therapy.

\section{Results}

The present study was done to evaluate whether low frequency Pulsed Electro Magnetic Field (PEMF) therapy is effective in patients with osteoarthritis knee using surface Electromyography (sEMG) of vastus medialis.

The mean age of the individuals included in the present study was $62.08 \pm 4.36$ years with the 50 to 70 years. The mean duration of symptoms of osteoarthritis in study subjects was $3.46 \pm 1.53$ years. The average BMI was found to be $27.57 \pm 2.59$.

Surface electromyography of the vastus medialis muscle was done in the study group and the amplitude was compared before and after Pulsed Electro Magnetic Field therapy. The mean values of amplitude are furnished in table 1

Table 1. Comparison of mean values of the amplitude of surface electromyography in vastus medialis before and after PEMF therapy.

\begin{tabular}{|c|c|c|c|}
\hline Variable & Group & Side & P-Value \\
\hline \multicolumn{2}{|c|}{ Amplitude of Before PEMF } & & $75.98 \pm 3.97$ \\
\hline $\begin{array}{l}\text { sEMG of } \\
\text { vastus }\end{array}$ & After PEMF & Right & $214.18 \pm 61.8<0.001^{* *}$ \\
\hline $\begin{array}{l}\text { medialis } \\
\text { (in } \mu \text { Volts) }\end{array}$ & $\begin{array}{l}\text { Before PEMF } \\
\text { After PEMF }\end{array}$ & Left & $\begin{array}{l}78.52 \pm 4.44 \\
253.06 \pm 69.5\end{array}<0.001 *$ \\
\hline
\end{tabular}

Table 1 shows that the mean amplitude of sEMG in vastus medialis was found to be significantly increased ( $p$ $<0.001$ ) in the study group following the Pulsed Electro Magnetic Field (PEMF) therapy.

\section{Discussion}

The present study revealed that Low frequency PEMF (10 $\mathrm{Hz}$ ) can be used as a treatment modality for Osteoarthritis. PEMF therapy, when given for a sufficient time (21 days) can improve the stiffness and the power of muscles as seen by an increase in amplitude of electromyography. Similar results are shown by various studies found in the literature. Fini et al stated that PEMF being applied at $75 \mathrm{~Hz}, 1.6 \mathrm{mT}, 6 \mathrm{hrs}$ per day for three months proved to prevent the development of osteoarthritis in aged guinea pigs [13]. Aaron and Ciombor et al examined the effects of PEMF in a decalcified bone matrix. The observations were noted with the increase in matrix synthesis which is stimulated by the proliferation of mesenchymal cells [14]. Ciombor et al had proved the enhanced synthesis of cartilage under the effect of themagnetic field applied at a burst of $4.5 \mathrm{~ms}$ duration and further repeated at 15 bursts [15]. De Mattei et al observed the anabolic effects of PEMF in the cartilage at different ranges of exposure length $(1,4,9$ and $24 \mathrm{~h})$, different frequencies $(2,37,75$ and $110 \mathrm{~Hz})$ and magnitudes $(0.5,1,1.5,2 \mathrm{mT})[16]$. Takahashi $\mathrm{K}$ et al had shown the effects of PEMF on the synthesis of bone. He stated that the osteogenesis was mainly due to the effect on osteoblasts and the DNA synthesis was enhanced by the exposure of PEMF at specific combinations of pulse width $25 \mu$ s, frequency $(10,100$ $\mathrm{Hz})$ and intensity $(2 \times 10-5$ and $8 \times 10-5 \mathrm{~T})$ [17]. Ganguly et al observed the effectiveness in reducing pain, stiffnes and improving function following PEMF therapy. He concluded that the patients with negative rheumatoid factor experience significant benefits from PEMF therapy [18].

\section{Conclusion}

The study shows that Low frequency PEMF $(10 \mathrm{~Hz})$ can be used as a treatment modality for Osteoarthritis. PEMF therapy, when given for a sufficient time (21 days) can improve the stiffness and the power of muscles as seen by the increase in amplitude of electromyography. Hence, PEMF, a novel approach, can be used in treating chronic osteoarthritis knee adjunctive to the pharmacotherapies that are currently in use.

Limitation of the study: Further studies including many diabetic and hypertensive participants and assessment of the efficacy of PEMF therapy by other markers of the disease should be done.

\section{Source of funding: Self}

\section{Conflict of Interest: Nil}

Acknowledgment: We acknowledge great help received from the scholars whose articles are cited and included in the references of this manuscript.

\section{References}

[1] Di Cesare P, Abramson S, Samuels J. Pathogenesis of osteoarthritis.In: Firestein GS, Kelley WN, eds. Kelley's Textbook of Rheumatology .8th ed. Philadelphia, Pa.: Saunders Elsevier; 2009: 1525-40. 
[2] Sharma MK, Swami HM, Bhatia V, Verma A, Bhatia SP, Kaur G. An Epidemiological Study of Correlates of Osteo-Arthritis in Geriatric Population of UT Chandigarh. Indian J Community Med.2007;32:77-8

[3] Parazzini F, Progretto Menopausa. Italia Study Group. Menopausal status, hormone replacement therapy use and risk of self-reported physiciandiagnosed osteoarthritis in women attending menopause clinics in Italy. Maturitas 2003;20;46:207-12

[4] Moskowitz RW. Osteoarthritis symptoms and signs. Osteoarthritis Diagnosis and Management; 5th edtition, WB Saunders 1993;255-261

[5] Hurley MV, Scott DL, Rees J, Newham DJ. Sensorimotor changes and functional performance in patients with knee osteoarthritis. Ann Rheum Dis. 1997;56(11):641-8

[6] Liikavainio T, Lyytinen T, Tyrväinen E, Sipilä S, Arokoski JP. Physical function and properties of quadriceps femoris muscle in men with knee osteoarthritis. Arch Phys Med Rehabil. 2008;89 (11):2185-94

[7] Diracoglu D, Baskent A, Yagci I, Ozcakar L, Aydin R. Isokinetic strength measurements in early knee osteoarthritis. Acta Reumatol Port.2009;34(1):72-7

[8] Ikeda S, Tsumura H, Torisu T. Age-related quadriceps-dominant muscle atrophy and incident radiographic knee osteoarthritis. J Orthop Sci. 2005; 10(2):121-6

[9] Petterson SC, Barrance P, Buchanan T, BinderMacleod S, Snyder-Mackler L.Mechanisms underlying quadriceps weakness in knee osteoarthritis. Med Sci Sports Exerc. 2008;40(3):422 $-7$

[10] Basmajian JV, De Luca CJ. Muscles Alive Their
Function Revealed by Electromyography. Williams Wilkins, Baltimore 1985 ISBN 0-683-00414-X

[11] Jari PA, Taru V,Markkuk, ölavi A: Activation atlumbar parsapinal and abdominal muscles during therapeutic exercises in chronic low back pain patients. Arch of Phy. Med. and Rehab 2004; 85:823

[12] Fischer G, Pelka RB, Barovic J: Adjuvant treatment of knee osteoarthritis with weak pulsing magnetic fields. Results of a placebo-controlled trial prospective clinical trial. Z Orthop Ihre Grenzgeb $2005 ; 143: 544-50$

[13] Fini M, Giavaresi G, Torricelli P, Cavani F, Setti S, Cane V \& Giardino R, Pulsed electromagnetic fields reduce knee osteoarthritic lesion progression in the aged Dunkin Hartley Guines pig, J Orthop Res. 2005;23:899

[14] Aaron RK \& Ciombor DMcK, Acceleration of experimental endochondral ossification by biophysical stimulation of the progenitor cell pool, J Orthop Res.1996;14:582.

[15] Ciombor DMcK, Aaron RK, wang S and Simon B, Modification of osteoarthritis by pulsed electromagnetic field - morphological study. Osteoarthritis and Cartilage.2003;11:455

[16] De Mattei M, Caruso A, Pezzetti F, Pellati A, Stabellini G, Sollazzo V \& Traina GC, Effects of pulsed electromagnetic fields on human chondrocyte proliferation, Connect Tissue Res.2001; 42:269

[17] Takahashi K, Kaneko I, Date M \& Fukada E, effect of pulsing electromagnetic fields on DNA synthesis in mammalian cell culture, Experientia.1986;42:185

[18] Ganguly KS, Sarkar AK, Datta AK \& Rakshit A, A study of the effects of pulsed electromagnetic field therapy with respect to serological grouping in rheumatoid arthritis, J Indian Med Asso. 1998;96:272. 